\title{
THE RELATIONSHIPS OF ANKLE LIGAMENTOUS INJURIES WITH PHYSICAL AND POSITIONAL CHARACTERISTICS IN COLLEGE FOOTBALL PLAYERS
}

\author{
SHINOBU NISHIMURA, KOICHI NAKAZATO and HIROYUKI NAKAJIMA
}

\begin{abstract}
The purpose of this study was to investigate the relationships of ankle ligamentous injuries with physical and positional characteristics in college football players. Specific hypothesis was addressed whether medial ankle ligamentous complex (MALC) injury was often caused by oversized players, offensive or defensive linemen.

53 subjects participated in this study. We examined their previous history of ankle ligamentous injuries and classified them into three groups: (1) the lateral ankle ligamentous complex (LALC) injury group; (2) the MALC injury group; and (3) the no ankle ligamentous (NAL) injury group. Regarding MALC injury, we obtained the following results. First, MALC injury occupied 35\% of total ankle ligamentous injuries. Second, both body weight and BMI of the MALC injury group were signi. ficantly the heaviest and largest of the groups. Lastly, OL, LB, and DL occupied highest occurring percentage $(75 \%)$ of MALC injury.

We concluded that MALC injury tended to be incurred by the players with heavier body weight and/or larger BMI. Players OL, LB, or DL also suffered MALC injury. Thus, these results suggest that body weight, BMI and position are considered as the risk factor for MALC injury.
\end{abstract}

(Jpn. J. Phys. Fitness Sports Med. 2004, $53: 281 \sim 292$ )

key word : Football, Ankle Ligamentous Injury, Physical Characteristics, Positional Characteristics

\section{Introduction}

American Football (football) is known as a collision sport with one of the highest incidence of sports injuries. Even though the football equipment (helmets and pads) has been improved in quality, many injury cases are still occurring. Injury cases of lower extremity, such as ankle and knee, are commonly seen in football. ${ }^{1,2)}$

With an estimate between 20 to 25 percent, ankle injuries are most frequently caused in running and jumping activities. ${ }^{3-5)}$ Ankle sprains also have a relatively high re-injury rate because the residual instability and loss of the joint's proprioception of position remain in its ligaments. ${ }^{4,5)}$ Lateral ankle ligamentous injuries are the most common injuries to the lower extremities in players due to the inversion ankle sprain. ${ }^{3,5 \sim 7)}$ In contrast, medial ankle and syndesmosis ligamentous injuries due to eversion and external rotation of lower extremity cause only five to ten percent of all ankle sprains. ${ }^{1,2,8)}$ Despite such low incidence, these injuries need a longer time for rehabilitation and recovery compared with lateral ankle ligamentous injury. ${ }^{8)}$ Also, although these injuries are considerably caused by football players, ${ }^{8,9)}$ nobody has reported on some factors to cause them enough.

The heavier the body weight is, the higher the risk of musculoskeletal injury will be. ${ }^{10)}$ In other words, it seems that medial ankle and syndesmosis ligamentous injuries as the lower extremity injury must be caused by heavy football players. However, no researchers have directly examined such a problem, even though these evidences clearly show that there are some relationships between medial ankle and syndesmosis ligamentous injuries and body weight. 
The purpose of this study was to investigate the relationships of ankle ligamentous injuries related to football activities, specifically during practice and games, based on the physical and positional characteristics of college football players. We especially hypothesized that medial ankle and syndesmosis ligamentous injuries would often be caused by oversized college football players, such as offensive or defensive linemen.

\section{Methodology}

\section{II-A. Subjects}

This study was done with the N University American Football team. The subjects included 53 players' physical characteristics were as follows: age $(20.2 \pm 1.5 \mathrm{y} / \mathrm{o})$; height $(174.9 \pm 5.7 \mathrm{~cm})$; body weight $(81.3 \pm 16.4 \mathrm{~kg})$; body mass index (BMI) $\left(26.2 \pm 4.1 \mathrm{~kg} / \mathrm{m}^{2}\right)$; and years of football experience $(4.7 \pm 1.6$ years $)$. The subjects were also divided by six positional groups : (1) offensive linemen ( $\mathrm{OL}: \mathrm{n}=7$ ); (2) running backs and quarterbacks ( $R B: n=13)$; (3) wide receivers and tight ends (WR: $n=15)$; (4) defensive linemen $(\mathrm{DL}: \mathrm{n}=5) ;(5)$ line backers $(\mathrm{LB}: \mathrm{n}=5)$; and (6) defensive backs (DB: $n=8)$.

\section{II-B. Data Collection}

The study consisted of three major parts : (1) medical checkup-/-preparticipation physical examination, (2) questionnaire, and (3) a man-to-man individual session. All data were collected by the NSSU American Football Team Golden Bears medical staff.

\section{II-B-1. Medical Checkup-/-Preparticipation Physical Examination}

The medical checkup-/-preparticipation physical examination was initially conducted in April of the players' freshman year. The points of assessment included (a) Physical characteristics, such as height, body weight, or BMI, (b) Previous history of injury related to football activities (Inspection), (c) the orthopedic screening examination (Palpation), and (d) the assessment of joint flexibility (Laxity tests). First point of the assessment, (a), was evaluated by the team athletic trainers, and last three points; (b), (c), and (d); were diagnosed by the team physician. The results of this assessment are also based on the N Uni. versity Football Individual Injury Report, and any kinds of injury/illness will be kept filing until graduation.

Regarding diagnosis of ankle ligamentous injuries, the following findings, such as point tenderness or symmetry, and the functional tests, such as range of motion or ligamentous testing, were the key to detect which ankle ligament was damaged. Table 1 showed in more detail. ${ }^{1,2,8}$, 12 14) The diagnosis was finally the charge of the team physician.

The laxity tests followed Nakajima's method ${ }^{15)}$ and checked the joint flexibility of six major joints (wrist, elbow, shoulder, hip, knee, and ankle) and spine. The laxity score expresses seven points in total and is calculated when each joint reaches the standard point decided.

\section{II-B-2. Questionnaire}

53 subjects with 4.7 years of average football experience conducted and completed the question. naire about their medical history of all ankle injuries related to football activities in May, 2001. An ankle injury was defined as an injury which resulted in the absence from practice and/or games for more than one week. Ankle injuries incurred by the subjects during the 2001 regular season were also added as injury cases to the questionnaire.

After collecting the questionnaires, the team athletic trainers checked all the ankle injuries carefully against the N University Football Individual Injury Report and made sure that there were no oversights. 
Table 1. Findings and Functional tests for Diagnosis of Ankle Ligamentous Injuries.

\begin{tabular}{|c|c|c|}
\hline Ligament & Findings & Functional tests \\
\hline \multirow{2}{*}{ Anterior Talofibular } & $\begin{array}{l}\text {-Point Tenderness around anterior-inferior part of lateral } \\
\text { malleolus of injured ankle. }\end{array}$ & $\begin{array}{l}\text {-Increasing pain with Plantar Flexion and Inversion of } \\
\text { iniured ankle. } \\
\text { (+) Anterior Drawer Test. }\end{array}$ \\
\hline & $\begin{array}{l}\text {-Swelling around lateral malleolus of injured ankle. } \\
\text {-Increasing tissue temperature around lateral malleolus of } \\
\text { iniured ankle. }\end{array}$ & \\
\hline Calcaneofibular & $\begin{array}{l}\text { - Point Tenderness around inferior part of lateral malleolus of } \\
\text { iniured ankle. } \\
\text {-Swelling around lateral malleolus of injured ankle. } \\
\text { - Increasing tissue temperature around lateral malleolus of } \\
\text { iniured ankle. }\end{array}$ & $\begin{array}{l}\text {-Increasing pain with Plantar Flexion and/or Inversion of } \\
\text { iniured ankle. } \\
\cdot(+) \text { Inversion Stress Test. }\end{array}$ \\
\hline Deltoid & $\begin{array}{l}\text {-Point Tenderness around inferior half part of medial malleolus } \\
\text { of iniured ankle. } \\
\text {-Swelling around medial malleolus of iniured ankle. } \\
\text {-Increasing tissue temperature around medial malleolus of } \\
\text { iniured ankle. }\end{array}$ & $\begin{array}{l}\text {-Increasing pain with Eversion and/or Plantar Flexion and/or } \\
\text { Dorsiflexion of iniured ankle. } \\
\cdot(+) \text { Eversion Stress Test and/or }(+) \text { External Rotation Test*. }\end{array}$ \\
\hline \multirow{2}{*}{ Anterior Inferior Tibiofibular } & $\begin{array}{l}\text {-Point Tenderness around anterior-superior parts of malleoli of } \\
\text { injured ankle. }\end{array}$ & \multirow{2}{*}{$\begin{array}{l}\text { Increasing pain with Dorsiflexion and External Rotation of } \\
\text { iniured ankle. } \\
\text { (+) External Rotation Test } \\
\text { ( }\end{array}$} \\
\hline & $\begin{array}{l}\text {-Swelling around anteriorsuperior parts of malleoli of iniured } \\
\text {-Increasing tissue temperature around anterior'superior parts of } \\
\text { malleoli of injured ankle. }\end{array}$ & \\
\hline
\end{tabular}

Referred to: Principles of Athletic Training ${ }^{1)}$, Sports Injuries ${ }^{2)}$, Sports Injury Managament ${ }^{8)}$, Evaluation of Orthopedic and Athletic Injuries ${ }^{12)}$, Orthopedic Physical Assessment ${ }^{13)}$, \& Concepts of Athletic Training ${ }^{14)}$.

* External Rotation Test is used to determine injury to the Deltoid or Anterior Inferior Tibiofibular ligaments, but the location of pain for the positive result is different.

— Deltoid ligament: Pain increase around inferior half part of medial malleolus of ankle.

L Anterior Inferior Tibiofibular ligament: Pain increase around anterior-superior parts of malleoli of ankle.

**All findings and functional tests were compared bilaterally.

\section{II-B-3. A Man-to-Man Individual Session}

A man-to-man individual session was held by the N University football athletic trainer for thirty minutes with each subject in July, 2001. The athletic trainer with certificates of National Athletic Trainers' Association and National Strength and Conditioning Association evaluated all players who recorded previous ankle injuries, and classified them according to the mechanism of injury and the results of the medical checkup and the questionnaire.

We used three data collecting methods (medical checkup, questionnaire, and individual session) above and classified the data with adopting manual testing as the functional test; however, only the testing might be not enough to diagnose. Thus, we won't positively mention which ankle ligament is sprained. We mainly discussed how the ankle ligamentous injuries were caused.

\section{II-C. Classification and Definition of Ankle Ligament}

There are three distinct ankle ligaments, the anterior and posterior talofibular ligaments and calcaneofibular ligament, at the lateral side of the ankle. ${ }^{16)}$ According to the anatomical lateral view of the ankle joint, this study defines two of the three ligaments, anterior talofibular ligament (ATFL) and calcaneofibular ligament (CFL), as the lateral ankle ligamentous complex (LALC). The mechanism of injury of medial ankle and syndesmosis ligaments, deltoid and anterior inferior tibiofibular ligaments, is similar. ${ }^{1,2,8)}$ The most common mechanism involves a player's lower leg that is forced to external rotation in the eversion position of the ankle joint. Thus, this study defines deltoid ligament (Del-L) and anterior inferior tibiofibular ligament (AITFL) as the medial ankle ligamentous complex (MALC).

\section{II-D. Statistical Analysis.}

All data results except laxity scores were 
analyzed using One-way ANOVA, and multiple comparison post test of Tukey was adopted for further analysis. Kruskal-Wallis test was used for comparisons of laxity scores, and Mann-Whitney $\mathrm{U}$ test was adopted as multiple comparison post test. A P value of 0.05 or less was used to define statistical significance.

This study was performed after informed consent was obtained from all players. The Ethics Committee approval from Nippon Sport Science University was granted to perform this study.

\section{Results}

III-A. Ankle Injury and Ankle Ligamentous Injury Related to Football Activities

25 of 53 subjects (47\%) suffered 53 cases of ankle injury related to football activities one or more times. The ankle injuries were classified into four groups: (1) Ligamentous injury, (2) Fracture, (3) Overuse injury, and (4) Others. The ligamentous injury group recorded the largest number of ankle injuries which was 34 cases
$(65 \%)$, and the remaining results were as follows: fracture group eight cases (15\%); overuse injury group six cases (11\%); and others group five cases (9\%) (Figure 1 ).

The 34 cases of ankle ligamentous injury were further categorized. From the point of view of the mechanism of injury, LALC and MALC injuries were caused $22(65 \%)$ and $12(35 \%)$ cases respectively. CFL injuries were caused $23 \%$ ( 8 cases) fol. lowed by ATFL injuries $21 \%$ ( 7 cases), ATFL \& CFL simultaneous injuries $21 \%$ ( 7 cases $)$, Del-L injuries $15 \%$ ( 5 cases), A ITFL injuries $15 \%$ ( 5 cases), and Del-L \& AITFL simultaneous injuries $5 \%(2$ cases) (Figure 2$)$.

\section{III-B. Three Ankle Ligamentous Injury Groups}

25 of 53 subjects suffered 34 cases of ankle ligamentous injury. We divided the subjects into three groups: (1) subjects who suffered LALC injury; (2) subjects who suffered MALC injury; and (3) subjects who suffered no ankle

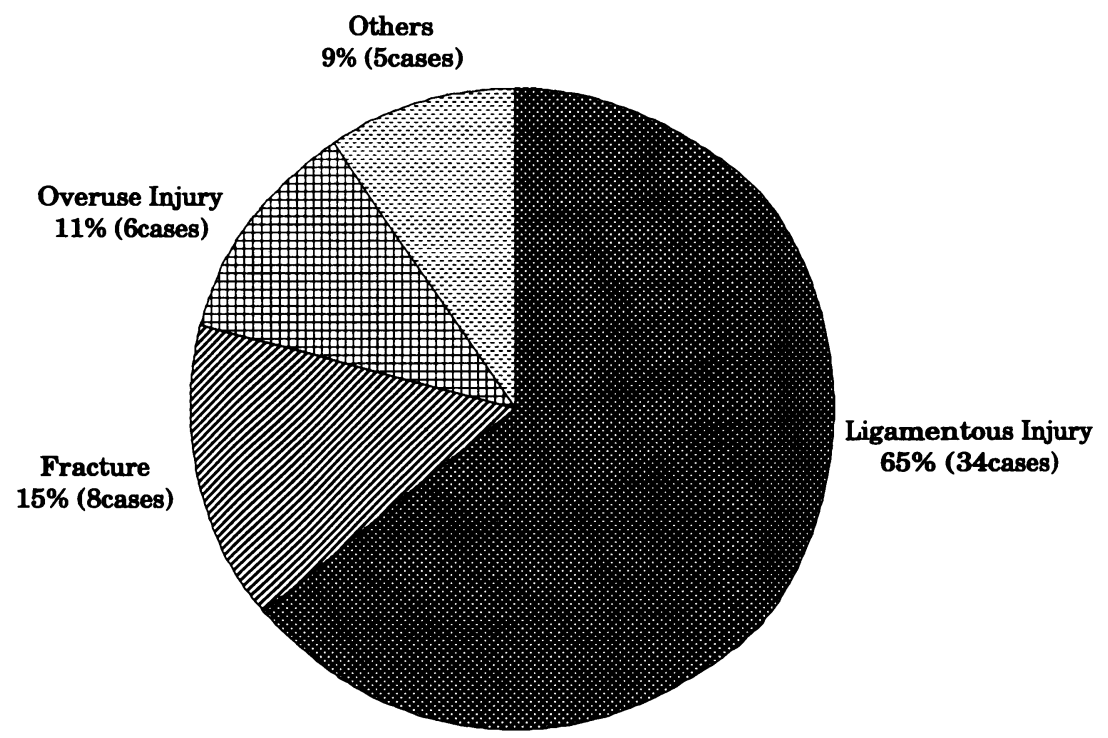

Figure 1. Ankle Injuries related to Football Activities (53 cases) 53 cases of ankle injuries related football activities were caused by 53 subjects during the last five years and the 2001 regular season. The ankle injuries were further classified into four groups: (1) Ligamentous Injury ; (2) Fracture ; (3) Overuse Injury ; and (4) Others. 


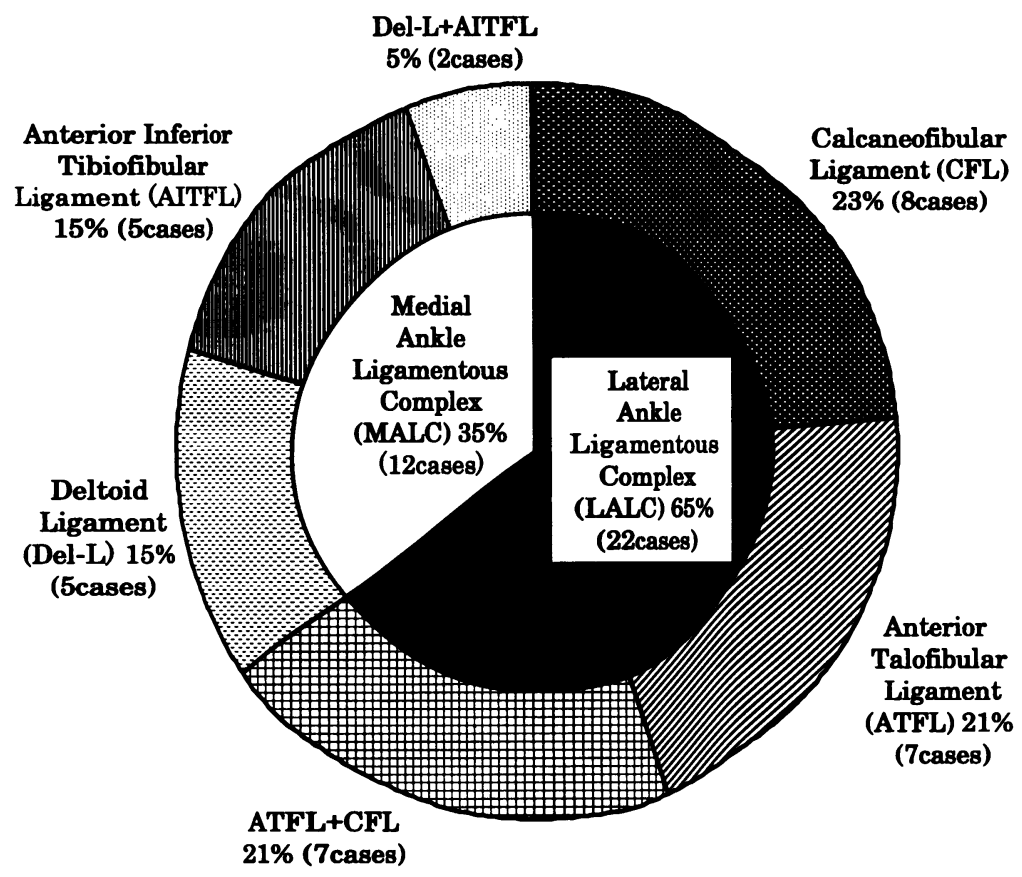

Figure 2. Ankle Ligamentous Injuries related to Football Activities (34 cases) 34 cases of ankle ligamentous injuries were classified based on the mechanism of injury: (1) Lateral Ankle Ligamentous Complex (LALC) injury (Anterior Talofibular Ligament (ATFL), Calcaneofibular Ligament (CFL), and ATFL \& CFL] and (2) Medial Ankle Ligamentous Complex (MALC) injury〔Deltoid Ligament (Del-L), Anterior Inferior Tibiofibular Ligament (AITFL), and Del-L \& AITFL].

ligamentous injury (NAL injury) (Table 2).

Eight of the 25 subjects who suffered both LALC and MALC injuries were categorized in each group. Also, one of the 25 subjects suffered two LALC injury cases, but we categorized him as one subject in the LALC injury group. Consequently, 21 subjects suffered 22 LALC injury cases, twelve subjects suffered twelve MALC injury cases, and 28 subjects suffer NAL injury cases.

Table 2. Comparison Between Physical Characteristics and Type of Ankle Ligamentous Injury Group.

\begin{tabular}{|c|c|c|c|c|c|c|c|}
\hline Type of Group & $\begin{array}{l}\text { Age } \\
(y / 0)\end{array}$ & $\begin{array}{c}\text { Height } \\
\text { (cm) }\end{array}$ & $\begin{array}{c}\text { Body } \\
\text { Weight } \\
\text { (ag) }\end{array}$ & $\begin{array}{c}\text { BMI } \\
\left(\mathbf{k g} / \mathbf{m}^{2}\right)\end{array}$ & $\begin{array}{c}\text { Years of } \\
\text { Football } \\
\text { Experience (yr) }\end{array}$ & $\begin{array}{l}\text { Laxity } \\
\text { Scores } \\
\text { (Point) }\end{array}$ & $\begin{array}{c}\text { Laxity Scores of } \\
\text { Ankle Joint } \\
\text { (Point) }\end{array}$ \\
\hline $\begin{array}{l}\text { Lateral Ankle Ligamentous Complex Injury } \\
\text { (LALC Injury) ( 21subjects*) }\end{array}$ & $20.7 \pm 1.6$ & $173.9 \pm 6.0$ & $\int^{79.2 \pm 16.1}$ & $26.1 \pm 4.6$ & $4.9 \pm 1.6$ & $1.9 \pm 1.2$ & $0.4 \pm 0.5$ \\
\hline $\begin{array}{l}\text { Modial Ankle Ligamentous Complex Injury } \\
\text { (MALC Injury) ( 12aubjecto") }\end{array}$ & $20.2 \pm 1.7$ & $175.7 \pm 5.4$ & $94.2 \pm 16.2$ & $30.4 \pm 4.5$ & $5.0 \pm 1.5$ & $1.4 \pm 0.9$ & $0.3 \pm 0.4$ \\
\hline $\begin{array}{l}\text { No Ankle Ligamentous Injury } \\
\text { (NAL Injury) ( 28-anjjects") }\end{array}$ & $20.0 \pm 1.3$ & $174.9 \pm 5.7$ & $80.5 \pm 15.8$ & $26.1 \pm 3.9$ & $4.6 \pm 1.6$ & $1.6 \pm 1.3$ & $0.1 \pm 0.2$ \\
\hline
\end{tabular}

$\dagger 1: p<0.05$ in One-way ANOVA (Tukey)

$\dagger 2: p<0.05$ in Kruskal-Wallis test (Mann-Whitney U test)

* 34 ankle ligamentous injury cases were caused by 25 of 53 subjects. 8 of the 25 subjects who suffered both LALC and MALC injuries were categorized in each group. Also, 1 of the 25 subjects suffered 2 LALC injury cases, but we categorized him as 1 subject in the LALC injury group. Consequently, 21 subjects suffered 22 LALC injury cases, 12 subjects suffered 12 MALC injury cases, and 28 subjects suffer NAL injury cases. 
Both body weight and BMI of the MALC injury group were significantly the heaviest and the largest of the groups $(p<0.05)$. Laxity scores of ankle joint of the NAL injury group were significantly the lowest of the groups $(p<0.05)$.

\section{III-C. Mechanism of Ankle Ligamentous Injury}

The 34 ankle ligamentous injury cases were classified into six groups based on the mechanism of injury (Table 3 ). The highest incidence of LALC injury in this study was CFL injury. CFL injury was caused by a subject's ankle being in a position of "inversion" when the subject either tried to change direction or land on the ground after jumping. ATFL injury was caused by a subject's ankle being in a position of "plantar flexion and inversion" when the subject sustained an opponent's tackle or block. Seven cases of ATFL and CFL simultaneous injuries were reported and were caused by the following mechanisms: the subject fell down on the ground with the ankle position of "plantar flexion and inversion" after sustaining a tackle or block similar to the ATFL injury. Furthermore, the subject's injured lower leg was forced to be in a position of "internal rotation" due to the opponent falling down on the injured ankle.

We also surveyed mechanism of MALC injury. Del-L injury was caused by a subject's ankle being in a position of "dorsiflexion" when the sub. ject sustained a tackle or block. Then, the opponent fell down on the subject's ankle from the lateral direction as the "valgus force" so that the ankle was forced to be in a position of "eversion." AITFL injury was caused by a subject's ankle being in a position of "dorsiflexion and eversion" similar to the Del-L injury. Moreover, the subject's injured lower leg was forced to be in a position of "external rotation" due to the opponent falling down on the subject's ankle. Lastly, Del-L and AITFL simultaneous injuries were caused by the "dorsiflexion, eversion, and external rotation" position similar to the AITFL injury.

\section{III-D. Ankle Injury and Ankle Ligamentous Injury by Position}

The 53 ankle injury cases were classified into

Table 3. Mechanism of Injury.

\begin{tabular}{|c|c|c|}
\hline Injured Ligament & Mechanism of Injury & Circumstance of Injury \\
\hline \multicolumn{3}{|c|}{ Lateral Ankle Ligamentous Complex (LALC) (22 cases) } \\
\hline $\begin{array}{l}\text { Anterior Talofibular Ligament } \\
\text { (ATFL) (7cases) }\end{array}$ & Plantar flexion + Inversion & $\begin{array}{l}\text { Being blocked (3 cases) } \\
\text { Blocking ( } 2 \text { cases) } \\
\text { Being tackled (1 case) } \\
\text { Tackling (1 case) }\end{array}$ \\
\hline $\begin{array}{l}\text { Calcaneofibular Ligament } \\
\text { (CFL) (8 cases) }\end{array}$ & Inversion & $\begin{array}{l}\text { Changing the direction (5 cases) } \\
\text { Stepping on the opponent's foot (3 cases) }\end{array}$ \\
\hline $\begin{array}{l}\text { ATFL + CFL } \\
\text { (7 cases) }\end{array}$ & Plantar flexion + Inversion + Internal Rotation & $\begin{array}{l}\text { Being tackled ( } 3 \text { cases) } \\
\text { Blocking ( } 2 \text { casea) } \\
\text { Tackling ( } 2 \text { casea) }\end{array}$ \\
\hline \multicolumn{3}{|c|}{ Medial Ankle Ligamentous Complex (MALC) (12 cases) } \\
\hline$\left(\begin{array}{c}\text { Deltoid Ligament } \\
(\text { Del-L) (5 cases) }\end{array}\right.$ & Dorsiflexion + Eversion & $\begin{array}{l}\text { Blocking (2 casea) } \\
\text { Being blocked (2 cases) } \\
\text { Being tackled (1 case) }\end{array}$ \\
\hline $\begin{array}{l}\text { Anterior Inferior Tibiofibular Ligament } \\
\text { (AITFL) (5 cases) }\end{array}$ & Dorsiflexion + Eversion + External Rotation & $\begin{array}{l}\text { Blocking (2 casea) } \\
\text { Being tackled (1 case) } \\
\text { Being blocked (1 case) } \\
\text { Tackling (1 case) }\end{array}$ \\
\hline $\begin{array}{l}\text { Del-L + AITFL } \\
(2 \text { cases })\end{array}$ & Dorsiflexion + Eversion + External Rotation & Being blocked (2 cases) \\
\hline
\end{tabular}

Total (34 cases*)

* 34 ankle ligamentous injury cases were caused by 25 of 53 subjects. 8 of the 25 subjects who suffered both LALC and MALC injuries were categorized in each group. Also, 1 of the 25 subjects suffered 2 LALC injury cases, but we categorized him as 1 subject in the LALC injury group. Consequently, 21 subjects suffered 22 LALC injury cases, 12 subjects suffered 12 MALC injury cases, and 28 subjects suffer NAL injury cases. 


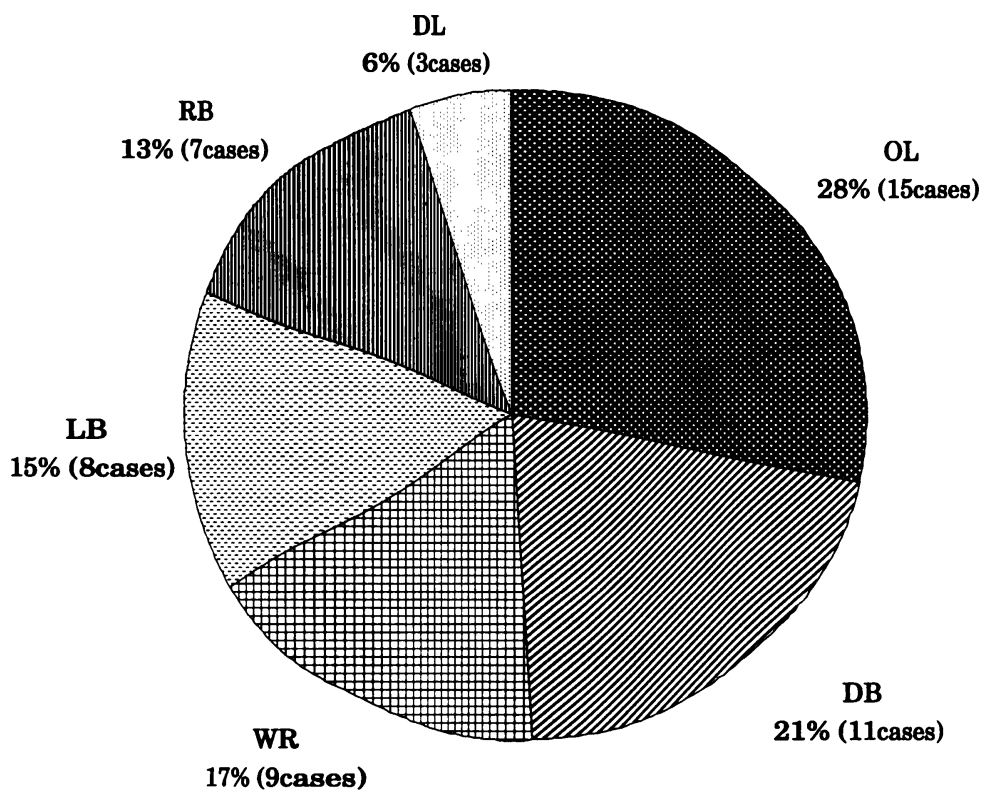

Figure 3. Ankle Injuries by Position (53 cases)

53 cases of ankle injuries related football activities were classified into six positional groups: (1) OL (Offensive Linemen, $n=7$ ); (2) RB (Running Backs \& Quarterbacks, $n=$ 13); (3) WR (Wide Receivers \& Tight Ends, $n=15)$; (4) DL (Defensive Linemen, $n=5$ ); (5) LB (Line Backers, $n=5$ ), and (6) DB (Defensive Backs, $n=8$ ).

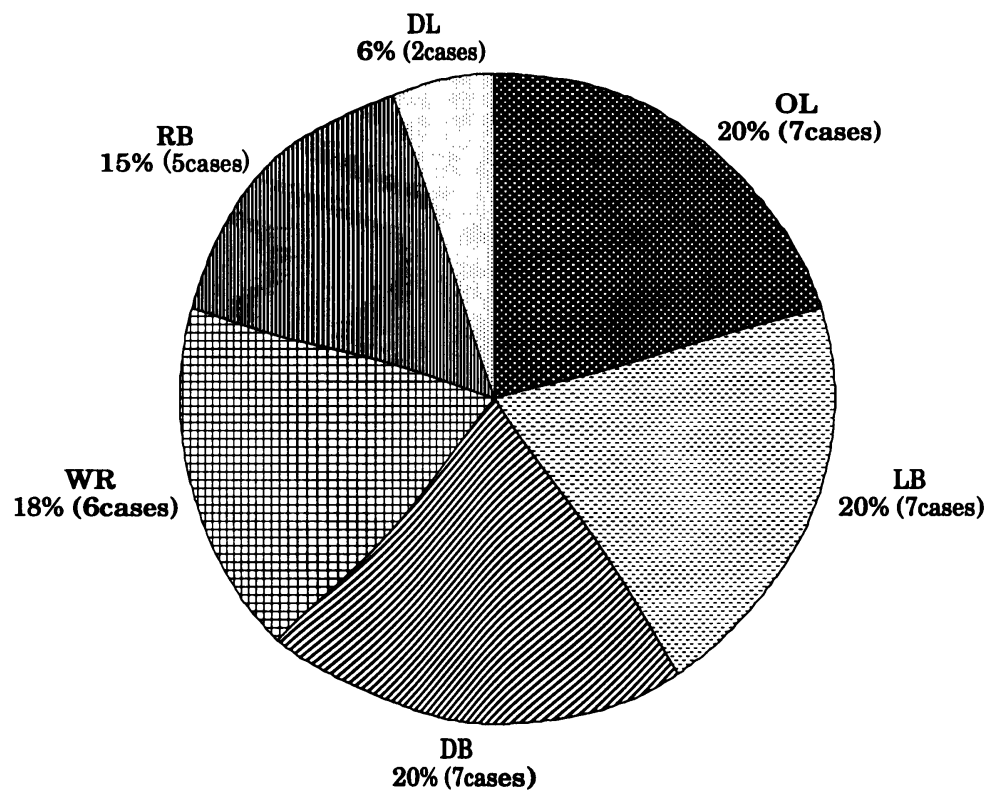

Figure 4. Ankle Ligamentous Injuries by Position (34 cases)

34 cases of ankle ligamentous injuries were classified into six positional groups: (1) OL (Offensive Linemen, $n=7$ ); (2) RB (Running Backs \& Quarterbacks, $n=13$ ); (3) WR (Wide Receivers \& Tight Ends, $n=15$ ): (4) DL (Defensive Linemen, $n=5$ ); (5) LB (Line Backers, $n=5$ ), and (6) DB (Defensive Backs, $n=8$ ). 
six positional groups (Figure 3). OL recorded the largest number of ankle injuries which was fifteen cases $(28 \%)$, and other positions' results were as follows : DB eleven cases (21\%), WR nine cases (17\%), LB eight cases (15\%), RB seven cases (13\%), and DL three cases (6\%).

Focusing on the 34 ankle ligamentous injury cases, the three positions of OL, LB, and DB occupied $60 \%$ of the total and recorded seven cases in each position (20\%) (Figure 4). OL suffered four MALC injury cases and the number was the largest of the positions (Table 4). RB suffered three ATFL and CFL simultaneous injuries which were the most of the positions. WR tended to experience the ATFL and CFL simultaneous injuries similar to $\mathrm{RB}$, but WR also suffered CFL injury caused as an intrinsic ankle ligamentous injury. DL did not experience any LALC injury cases, but two MALC injury cases were reported. LB experienced a pattern of ankle ligamentous injury similar to OL. DB most frequently experienced the LALC injury for a total of six cases.

According to Table 5, these statistical significances were found. Both body weight and BMI of

Table 4. Classification of Injured Ligament and Position.

\begin{tabular}{|c|c|c|c|c|c|c|}
\hline \multirow{2}{*}{ Injured Ligament } & \multicolumn{6}{|c|}{ Position } \\
\hline & OL & $\mathbf{R B}$ & WR & DL & LB & DB \\
\hline Lateral Ankle Ligamentous Complex (LALC) (22 cases) & 3 & 4 & 5 & $\mathbf{0}$ & 4 & 6 \\
\hline Anterior Talofibular Ligament (ATKL) (7 cases) & 2 & 1 & 1 & & 2 & 1 \\
\hline Calcaneofibular Ligament (CFL) (8 canes) & 1 & & 2 & & 2 & 3 \\
\hline ATYL + CFL (7 canes) & & 3 & 2 & & & 2 \\
\hline Medial Ankle Ligamentous Complex (MALC) (12 cases) & 4 & 1 & 1 & 2 & 3 & 1 \\
\hline Doltoid Ligament (Dot-L) (5 cases) & 2 & 1 & & & 2 & \\
\hline Anterior Inferior Tibiofibular Ligament (ATTKL) (5 cases) & $\mathbf{2}$ & & 1 & 1 & & 1 \\
\hline Del-L + ATTFL (2 cases) & & & & 1 & 1 & \\
\hline TOTAL (34 cases*) & 7 & 5 & 6 & 2 & 7 & 7 \\
\hline
\end{tabular}

*34 ankle ligamentous injury cases were caused by 25 of 53 subjects. 8 of the 25 subjects who suffered both LALC and MALC injuries were categorized in each group. Also, 1 of the 25 subjects suffered 2 LALC injury cases, but we categorized him as 1 subject in the LALC injury group. Consequently, 21 subjects suffered 22 LALC injury cases, 12 subjects suffered 12 MALC injury cases, and 28 subjects suffer NAL injury cases.

Table 5. Comparison Between Physical Characteristics and Position.

\begin{tabular}{|c|c|c|c|c|c|c|c|}
\hline $\begin{array}{c}\text { Position } \\
\text { (* of subjects*) }\end{array}$ & $\begin{array}{l}\text { Age } \\
\text { (y/o) }\end{array}$ & $\begin{array}{l}\text { Height } \\
\text { (cm) }\end{array}$ & $\begin{array}{c}\text { Body } \\
\text { Weight } \\
\text { (kg) }\end{array}$ & $\frac{B M I}{(\mathbf{k g} / \mathbf{m} 2)}$ & $\begin{array}{l}\text { Years of Football } \\
\text { Experience (yr) }\end{array}$ & $\begin{array}{l}\text { Laxity Scores } \\
\text { (Point) }\end{array}$ & $\begin{array}{l}\text { Laxity Scores of } \\
\text { Antle Joint (Point) }\end{array}$ \\
\hline OL $(n=7)$ & $20.1 \pm 0.7$ & $179.6 \pm 5.7$ & ${ }^{n} 108.4 \pm 8.0$ & ${ }^{n} 33.6 \pm 2.0$ & $5.1 \pm 2.0$ & $0.9 \pm 0.8$ & $0.1 \pm 0.4$ \\
\hline$R B(n=13)$ & $20.0 \pm 1.6$ & $172.5 \pm 6.0$ & $1273.4 \pm 5.9$ & $=24.7 \pm 2.0$ & $4.2 \pm 1.4$ & $1.9 \pm 1.4$ & $0.1 \pm 0.2$ \\
\hline WR $(n=15)$ & $20.5 \pm 1.5$ & $173.7 \pm 6.1$ & $71.5 \pm 10.0$ & ${ }^{12} 23.6 \pm 2.1$ & $4.5 \pm 1.5$ & $2.0 \pm 1.1$ & $0.2 \pm 0.4$ \\
\hline DL $(n=6)$ & $19.4 \pm 0.9$ & $179.0 \pm 2.9$ & ${ }^{n} 104.4 \pm 10.9$ & ${ }^{n} 32.6 \pm 3.5$ & $4.0 \pm 2.1$ & $0.7 \pm 0.6$ & $0.0 \pm 0.0$ \\
\hline LB $(n=5)$ & $21.2 \pm 2.3$ & $176.6 \pm 4.4$ & ${ }^{t 8} 87.6 \pm 5.4$ & ${ }^{* 8} 28.1 \pm 1.7$ & $5.2 \pm 1.8$ & $1.9 \pm 0.8$ & $0.7 \pm 0.5$ \\
\hline DB $(n=8)$ & $20.0 \pm 1.3$ & $173.1 \pm 3.1$ & $1270.4 \pm 2.4$ & ${ }^{123.5 \pm 0.4}$ & $5.5 \pm 1.5$ & $1.9 \pm 1.6$ & $0.3 \pm 0.5$ \\
\hline
\end{tabular}

$p<0.05$ in One-way ANOVA. (Tukey)

$\dagger 1$ : Body weight and BMI of OL \& DL were significantly heavier and larger than those of RB, WR, LB, \& DB.

$\uparrow 2$ : Body weight and BMI of RB, WR, \& DB were significantly lighter and smaller than those of OL, DL, \& LB.

$\dagger 3$ : Body weight and BMI of LB were significantly heavier and larger than those of RB, WR, \& DB.

$\uparrow 3$ : Body weight and BMI of LB were significantly lighter and smaller than those of OL \& DL.

* 53 subjects were divided by six positional groups : (1) OL (Offensive Linemen, $\mathrm{n}=7$ ) ; (2) RB (Running Backs \& Quarterbacks, $n=13$ ) ; (3) WR (Wide Receiver \& Tight Ends, $n=15):(4)$ DL (Defensive Linemen, $n=5)$; (5) LB (Line Backers, $\mathrm{n}=8$ ) ; and (6) DB (Defensive Backs, $\mathrm{n}=8$ ). 
OL and DL were significantly heavier and larger than those of RB, WR, LB, and DB $(p<0.05)$. Both body weight and BMI of LB were not only significantly heavier and larger than those of RB, WR, and DB, but were also significantly lighter and smaller than those of OL and DL $(p<0.05)$.

\section{Discussion}

53 subjects participated in this study, and 25 of the 53 subjects suffered 53 ankle injury cases related to football activities, including 34 ankle ligamentous injury cases. Some subjects often suffered ankle re-injuries, but they returned to play in less than one week with ankle taping or an ankle brace. We classified the 34 ankle ligamentous injury cases into three groups: (1) LALC; (2) MALC ; and (3) NAL injuries, and confirmed the following findings in this study.

\section{IV-A. Types of Ankle Ligamentous Injury in Football}

LALC injury was the most common injury in this study and occupied 65\% (22 cases) of the total ankle ligamentous injuries (Figure 2). In other words, $35 \%$ ( 12 cases) of MALC injury occurred in this study. Arnheim and Prentice ${ }^{1)}$ (2000) and $\mathrm{Fu}$ and Store ${ }^{2)}$ (2001) reported that MALC injury occurred in only five to ten percent of all ankle sprains; however, the results from this study revealed that MALC injury occurred three times more often during football activities. Thus, MALC injury more often tends to be caused in college football players, and this study supported the results of Anderson et al. ${ }^{8)}$ (2000) and Baytin et al. $^{9)}(1991)$.

\section{IV-B. Relationships of Ankle Ligamentous Injuries with Laxity Scores}

There was no significant difference in total laxity scores of three ankle ligamentous injury groups; however, laxity scores of ankle joint of the NAL injury group were significantly the smallest of the groups $(p<0.05)$ (Table 2). Both LALC and MALC injuries were indeed caused by a subject with larger laxity scores of ankle joint. Kubo$\mathrm{ta}^{17)}$ (1988) reported that total laxity scores of college football players with ligamentous injuries, 1.65 , were significantly larger than those without ligamentous injuries, 1.22. We divided the 53 subjects into two groups: (1) subjects who suffered LALC and/or MALC injuries (ankle ligamentous injury group) and (2) subjects who suffered neither LALC nor MALC injuries (no ankle ligamentous injury group), and compared the physical characteristics of those groups. We found out that no significant differences between the two groups except laxity scores of ankle joint. Total laxity scores of the ankle ligamentous injury group, 1.78, were not significantly larger than those of the no ankle ligamentous injury group, 1.59 , which didn't support the report of Kubota ${ }^{17)}$ (1988). Thus, we considered that total laxity scores might be not associated with the factor of ankle ligamentous injury in this study, but laxity scores of ankle joint significantly seemed to be the prediction of ankle ligamentous injury occurrence.

We found out that football players with larger laxity scores of ankle joint tended to cause LALC and/or MALC injuries in this study. From the following paragraph, we will focus on MALC injury and discuss it for further details.

\section{IV-C. Relationships of MALC Injury with Physical Characteristics}

Both body weight and BMI of the MALC injury group were significantly the heaviest and the largest of the groups $(p<0.05)$ (Table 2). Comparison of the MALC injury group and the other two groups in average body weight showed a difference of over ten kilograms. As a whole, sixteen of 53 subjects $(30 \%)$ were over $90 \mathrm{~kg}$ of body weight, and eight of the sixteen subjects $(50 \%)$ 
suffered MALC injury. In the MALC injury group, eight of twelve subjects (67\%) were over $90 \mathrm{~kg}$ of body weight. This result stood for the report of Kaplan et al. ${ }^{18)}$ (1995) that a player with over 90 $\mathrm{kg}$ of body weight was associated with a 2.5 times higher relative risk of injury.

Also, Tsuji and Inoue ${ }^{19)}$ (1998) mentioned and defined obesity as a person who is over 26.4 $\mathrm{kg} / \mathrm{m}^{2}$ of BMI. As a whole, 22 of 53 subjects $(42 \%)$ were over the standard $26.4 \mathrm{~kg} / \mathrm{m}^{2}$, and ten of the 22 subjects (45\%) sustained MALC injury. In the MALC injury group, ten of twelve subjects (83\%) were over $26.4 \mathrm{~kg} / \mathrm{m}^{2}$. Gomez et al. ${ }^{11)}$ (1998) mentioned that larger BMI was associated with increased rates of lower extremity injury among football linemen. Thus, our hypothesis was amply supported by these results that a subject with both heavier body weight and larger BMI undoubtedly tends to incur MALC injury.

\section{IV-D. Relationships of MALC Injury with Positional Characteristics}

The highest incidence of ankle ligamentous injury per position occurred in "OL, LB, and DB" with seven cases each in this study (Table 4). Focusing on MALC injury, the breakdown of the total of twelve MALC injury cases in positional order was as follows: OL four cases (33\%); LB three cases (25\%); DL two cases $(17 \%)$, and RB-/-WR-/-DB one case each (8\%) (Table 4).

These results suggest that there are some relationships of MALC injury with positional char. acteristics. As identified in Table 5, both body weight and BMI of OL, LB, and DL were significantly heavier and larger than players in the other positions; in addition, they occupied the highest occurring percentage (75\%) of MALC injury. In the three positions, there were sixteen of seventeen subjects (94\%) who were over $90 \mathrm{~kg}$ of body weight and/or over $26.4 \mathrm{~kg} / \mathrm{m}^{2}$ of BMI, and nine of the sixteen subjects (56\%) suffered MALC injury. More than one out of two subjects experienced it. Thus, both heaver body weight and larger BMI might be associated with increased rates of MALC injury among subjects who play in $\mathrm{OL}, \mathrm{LB}$, or DL, and these results upheld our hypothesis.

Regarding each position in detail, OL sustained four MALC injury cases, the highest number. While OL blocks an opponent, a team mate or another opponent falls down on him from the side as a valgus force. Then, the injured ankle of $\mathrm{OL}$ is in a position of "dorsiflexion" and is sometimes shifted to the "external rotation" of the lower leg. For that reason, $\mathrm{OL}$ might experience the greatest number of MALC injury in this study.

According to the role of $\mathrm{RB}$, a player carries the ball toward the end zone so that all opponents chase and tackle him every time. LALC injury is caused when the opponents fall down on the in jured ankle. Then, the injured ankle is placed in a position of "plantar flexion and inversion" while being forced to "internal rotation" of the injured lower leg. Consequently, RB experienced three ATFL \& CFL simultaneous injuries which were the highest number in all positions.

Two ATFL \& CFL simultaneous injuries to WR were caused similar to $\mathrm{RB}$ while being tackled; however, two CFL injuries were caused by a com. pletely different type of mechanism. The mechanism of CFL injury is that WR inverted his ankle as trying to change the running pace or the direction quickly in order to evade the opponent, or stepping on the opponent's foot after struggling to catch the ball in the air. For those reasons, WR might suffer solo CFL injury.

DL had the lowest incidence of ankle ligamentous injury. No LALC injury cases were reported; however, two MALC injury cases were reported. DL frequently sustains the block from the lateral side as well as from the front. Thus, DL suffered MALC injury similar to OL. 
LB also experienced a similar ankle ligamentous injury pattern to $\mathrm{OL}$. The role of $\mathrm{LB}$ is to tackle the ball carrier and to cover the middle defensive zone against passing offense. However, LB not only tackles the ball carrier but is also blocked by all opponents every time. LB has frequent opportunities to make contact against either the ball carrier or the blocker, causing an increase in the incidence of MALC injury. Also, solo CFL injury is caused by changing the pace and direction quickly while chasing WR. Thus, the incidence of ankle ligamentous injury increases.

DB experienced a similar ankle ligamentous injury pattern to WR. This position reported seven LALC injury cases and that number was the highest. The position's role is to tackle the ball carrier and to cover the deep defensive zone against passing offense. DB has to follow WR in the passing situation and to tackle him. Thus, DB suffers solo CFL injury due to the quick change of pace or direction or ATFL \& CFL simultaneous injuries due to the opponent falling down on the player's ankle after tackle.

Overall, the study associated with MALC injury hasn't been investigated thoroughly even though MALC injury is well known to be caused by football players. ${ }^{1,2,8,9)}$ We reported only 12 MALC injury cases, but it accounted for $35 \%$ of the total ankle ligamentous injuries in this retrospective study. The number is an exceedingly high number compared with other sports activities. We also suggest that the subjects with over $90 \mathrm{~kg}$ of body weight and/or $26.4 \mathrm{~kg} / \mathrm{m}^{2}$ of BMI who play in OL, $\mathrm{LB}$, or DL are likely to be suffered MALC injury. The physical characteristics are considered as the risk factors. However, we couldn't compare MALC injury with each position due to the insufficient number for analyzing the statistical significant. Therefore, we should keep investigating this as the further study.

\section{Conclusion}

This study investigated the relationships of ankle ligamentous injuries related to football activities with physical and positional characteristics. We summarize this study with the following conclusions.

1) Laxity scores of ankle joints of the NAL injury group were significantly the smallest of the groups $(p<0.05)$.

2) 34 cases of ankle ligamentous injury were caused in 53 college football players, and the details were as follows: LALC injury 22 cases (ATFL 7 cases, CFL 8 cases, and ATFL \& CFL 7 cases) and MALC injury 12 cases (Del-L 5 cases, AITFL 5 cases, and Del-L \& AITFL 2 cases). MALC injury occupied 35\% of the total ankle ligamentous injuries.

3) Both body weight and BMI of the MALC injury group were significantly the heaviest and the largest of the groups $(p<0.05)$.

4) OL, LB, \& DL occupied the highest occurring percentage $(75 \%)$ of MALC injury.

We concluded that MALC injury tended to be incurred by the players with heavier body weight and/or larger BMI. Players OL, LB, or DL also suffered MALC injury. Thus, these results suggest that body weight, BMI, and positions are considered as the risk factor for MALC injury.

\section{Acknowledgments}

We greatly appreciate the cooperation, understanding, and kindness of the NSSU American Football team Golden Bears coaching and medical staffs.

(Accepted Mar. 5, 2004)

\section{References}

1) Arnheim, D. D. \& Prentice, E. W., Principles of Athletic Training, Tenth Edition, McGraw-Hill Companies, Boston: MT,(2000), 446-514.

2) Fu, H. F. \& Stone, A. D., Sports Injuries, $2^{\text {nd }}$ Edition, Lippincott Willams \& Wilkins, Philadelphia: PA, (2001), 436-453. 
3) Kinzey, J. S., Ingersoll, D. C., \& Knight, L. K., The effects of selected ankle appliances on postural control, Journal of Athletic Training, (1997), 32, 300-303.

4) Karlsson, J. \& Andreasson, O. G., The effect of external ankle support in chronic lateral ankle joint instability, American Journal of Sports Medicine, (1992), 20, 257-261.

5) Karlsson, J., Sward, L., \& Andreasson, O. G., The effect of taping on ankle stability, Journal of Sports Medicine,(1993), 16, 210-215.

6) Isakov, E. \& Mizrahi, J., Is balance impaired by re. current sprained ankle?, British Journal of Spots Medicine, (1997), 31, 65-67.

7) Vaes, P., De Boeck, H., Handelberg, F., \& Opdecam, $P$., Comparative radiologic study of the influence of ankle joint bandages on ankle stability, American Journal of Sports Medicine,(1985), 13, 46-50.

8) Anderson, K. M., Hall, J. S., \& Martin, M., Sports Injury Management, Lippincott Williams \& Wilkins, Baltimore: Maryland, (2000), 483-528.

9) Baytin, F. M., Fischer, A. D., \& Newmann, L., Syndesmotic Ankle Sprains, American Journal of Sports Medicine,(1991), 19, 294-298.

10) Kaplan, A. T., Obesity in A High School Football Candidate: A Case Presentation, Medicine \& Science in Sports \& Exercise, (1992), 24, 406-409.

11) Gomez, E. J., Ross, K. S., Calmbach, L, W., Kimmel, B. R., Schmidt, R. D., \& Dhanda, R., Body Fatness and Increased Injury Rates in High School Football
Linemen, Clinical Journal of Sports Medicine, (1998), 8, 115-120.

12) Starkey, C. \& Ryan, J., Evaluation of Orthopedic and Athletic Injuries, F. A. Davis Company, Phi. ladelphia: PA,(1996), 86-126.

13) Magee, J. D., Orthopedic Physical Assessment, 3rd Edition, W. B. Saunders Company, Philadelphia: PA, (1997), 599-672.

14) Pfeiffer, P. R. \& Mangus, C. B., Concepts of Athletic Training, 2nd Edition, Jones and Bartlett Pub. lishers, Sudbury: MA, (1998), 208-225.

15) Nakajima, H., Undou no tameno Medical Check -Orthopedic course- (In Japanese), The Journal of Japanese Society of Clinical Sports Medicine, (2000), 17, 350-356.

16) Moore, L. K., Clinical Oriented Anatomy, Third Edition, Williams \& Wilkins, Baltimore: MD, (1992), 373-500.

17) Kubota, K., American Football ni okeru Team Doc. tor (Orthopedist) no Yakuwari -Medical Check wo chuushin toshite-(In Japanese), Japanese Journal of Sports Science,(1988), 7, 780-784.

18) Kaplan, A. T., Digel, L. S., Scavo, A. V., \& Arellana, B. S., Effect of Obesity on Injury Risk in High School Football Players, Clinical Journal of Sports Medicine,(1995), 5, 43-47.

19) Tsuji, M.\& Inoue, S., Genryou wo meguru Igaku -Saikin no Shinpo-, a. Genryou to Shokuji (In Japanese). The Journal of Clinical Sports Medicine, (1998), 15, 455-460. 